\title{
Identification of photocrosslinking peptide ligands by mRNA display
}

Yuteng Wu ${ }^{[a, b]}$ M. Teresa Bertran, ${ }^{[b]}$ Dhira Joshi, ${ }^{[c]}$ Sarah L. Maslen, ${ }^{[d]}$ Catherine Hurd, ${ }^{[a, e]}$ and Louise J. Walport*[a,b]

a. Protein-Protein Interaction Laboratory, The Francis Crick Institute, London NW1 1AT, United Kingdom

b. Department of Chemistry, Molecular Sciences Research Hub, Imperial College London, London W12 OBZ, United Kingdom

c. Peptide Chemistry, The Francis Crick Institute, London NW1 1AT, United Kingdom

d. Proteomics, The Francis Crick Institute, London NW1 1AT, United Kingdom

e. Crick-GSK Biomedical LinkLabs, GlaxoSmithKline, Gunnels Wood Road, Stevenage, SG1 2NY, United Kingdom

*Email address: I.walport@imperial.ac.uk

\begin{abstract}
Photoaffinity labelling is a promising method for studying protein-ligand interactions. However, obtaining a specific crosslinker can require significant optimisation. We report a novel mRNA display strategy, photocrosslinking-RaPID (XL-RaPID), and exploit its ability to accelerate the discovery of cyclic peptides that photocrosslink to a target of interest. As a proof of concept, we generated a benzophenone-containing library and applied XL-RaPID screening against a model target, the second bromodomain of BRD3. This crosslinking screening gave two optimal candidates that selectively labelled the target protein in cell lysate. Overall, this work introduces direct photocrosslinking screening as a versatile technique for identifying covalent peptide ligands from mRNA display libraries incorporating reactive warheads.
\end{abstract}

\section{Introduction}

Photoaffinity probes are powerful reagents for studying complex biological interactions. They can be used to identify new drug targets/off-target interactions, ${ }^{1-4}$ to uncover the structure and location of drug binding sites, ${ }^{5,6}$ for covalent protein labelling or for selective protein immobilisation. ${ }^{7-9}$ Photoaffinity probes typically consist of a photoactivatable functionality appended to a targetselective ligand. Upon exposure to light, the photoactivatable moiety irreversibly crosslinks to the target of interest. ${ }^{10} \mathrm{~A}$ variety of chemistries can be used for the photoactivatable moiety, including benzophenones (Bpa), diazirines and aryl azides. ${ }^{4,11-13} \mathrm{Bpa}$ is frequently used, due to its stability, 
relatively mild activation conditions ( $365 \mathrm{~nm}$ light), and low reactivity with water. ${ }^{14,15}$ In addition to its use in probes, an amino acid containing Bpa, $p$-benzoyl-L-phenylalanine ( $p \mathrm{Bpa}$ ), has been successfully incorporated into proteins through genetic code reprogramming to study protein-protein interactions in vitro and in vivo. ${ }^{16,17}$

The design and development of photoaffinity ligands for a specific target is not trivial. Current methods often rely on rational design using existing ligands and information on their target binding mode. ${ }^{5,12,18,19}$ This approach frequently results in low photocrosslinking efficiency, even when basing the probe on a high affinity ligand. A more efficient discovery approach would involve a method to directly screen large numbers of compounds for their ability to photocrosslink to a target of interest. In vitro display technologies such as mRNA display and phage display are ideal for this purpose as they allow the generation and screening of large, randomised libraries of peptides incorporating unnatural side chain chemistries. ${ }^{20}$ For example, such libraries have recently been applied to the development of photoswitchable ligands through incorporation of an azobenzene group, ${ }^{21}$ as well as the identification of covalent inhibitors by introducing reactive electrophilic warheads. ${ }^{22}$ The RaPID (random non-standard peptide integrated discovery) system is particularly well suited to this due to the ability to incorporate a wide variety of unnatural chemistries into peptides using flexible in vitro translation (FIT). ${ }^{20,23,24}$

Here, we report an mRNA display strategy, photocrosslinking-RaPID (XL-RaPID), to evolve peptides that undergo efficient covalent modification with their target of choice upon light activation (Figure 1). Having first adapted the affinity panning step to isolate only covalent target-binding peptides, we applied XL-RaPID screening against a model target, the second bromodomain of BRD3 (BRD3-BD2). Our XL-RaPID screen yielded efficient photocrosslinkers that selectively labelled the target protein in cell lysate.

\section{Results and discussion}

Our study began with the incorporation of the Bpa moiety into our mRNA displayed cyclic peptide library through including $p \mathrm{Bpa}$ in the FIT system. We synthesised the photoactivatable amino acid protected with a cyanomethyl ester (CME) group using previously reported procedures. ${ }^{25,26}$ The CME leaving group facilitated efficient charging onto a model tRNA (microhelix RNA, FAM-MiHx_23b) through eFx mediated aminoacylation (Supplementary Figure S1). Following charging onto an elongator tRNA (tRNA ${ }^{A s n}{ }_{C A U}$ ) to genetically reprogramme the methionine codon, $p$ Bpa was successfully incorporated into a model cyclic peptide by in vitro translation using a Met-free FIT system (PURExpress $^{\mathrm{TM}}, \Delta \mathrm{aa}, \triangle \mathrm{tRNA}, \mathrm{NEB}$ ) (Supplementary Figure S2). 
Next, we set out to modify the standard RaPID protocol to bias it towards the selection of photocrosslinking ligands. To retain only covalently crosslinked peptides during affinity panning, we envisaged replacing the standard wash buffers with wash buffers that would denature the target protein (Figure 2). ${ }^{23}$ This should allow removal of all non-covalently bound peptides, after which the crosslinked protein-peptide conjugates could be eluted from the beads by boiling. The DNA associated with the crosslinking peptides could then be isolated by PCR and carried forward to the next round. To avoid also retaining peptide sequences that crosslink to the immobilised streptavidin on the beads, or the beads themselves, we planned to mix the translated peptide library and biotinylated target protein and expose to UV irradiation to promote crosslinking in solution prior to target immobilisation on streptavidin beads.

To test the feasibility of our proposed strategy, we first confirmed that treating biotinylated proteinbound beads with guanidine chloride (up to $8 \mathrm{M}$ ) did not disrupt the interaction between biotin and streptavidin (Supplementary Figure S3). Subsequently, we used a model mRNA template encoding a previously identified non-covalent peptide ligand (3.1B) of the first bromodomain of BRD3, BRD3$\mathrm{BD} 1,{ }^{27}$ to confirm the effectiveness of our denaturing washes in removing non-covalent ligands. Pleasingly, after completing a mock selection round, whilst 3.9\% of the input DNA was recovered following standard affinity panning, almost no input DNA $(0.0069 \%)$ was recovered following washes with $5 \mathrm{M}$ guanidine (Supplementary Figure S4). This confirmed that the guanidine washes were sufficient to dissociate a tight binding peptide $\left(K_{D}=0.49 \mathrm{nM}\right)$ from its target protein ${ }^{27}$, most likely due to target denaturation.

Having optimised the individual steps of our XL-RaPID methodology, we set out to apply it to a model system. We aimed to identify photocrosslinking peptide ligands for the second bromodomain (BD2) of BRD3 from the bromodomain and extraterminal domain (BET) family of transcriptional coregulators, ${ }^{28-30}$ against which we have recently identified a series of potent non-covalent cyclic peptides. ${ }^{27}$ We constructed an mRNA displayed peptide library ( $>10^{12}$ members) containing six to twelve random amino acids, flanked by an initiator codon and a $C$-terminal cysteine prior to a GSGSGSlinker. Peptides were initiated with $N$-chloroacetyl-D-tyrosine (by flexizyme-mediated reprogramming of the start codon), to produce macrocyclic peptides through reaction with the cysteine. Internal positions coding for methionine were reprogrammed to introduce $p \mathrm{Bpa}$, resulting in a fraction (approximately $28 \%$ ) of the library having crosslinking capabilities. We envisaged that through iterative rounds of selection, only sequences containing at least one $p B p a$ would be enriched. The library was then subjected to five rounds of selection against BRD3-BD2 using our optimised XL-RaPID procedure. The peptide library and biotinylated BRD3-BD2 were first exposed to UV light $(365 \mathrm{~nm})$ for 
30 minutes at $0{ }^{\circ} \mathrm{C}$ to promote crosslinking. Following protein capture by magnetic streptavidin beads, stringent $5 \mathrm{M}$ guanidine washes $(2 \times 20 \mathrm{~min}$ ) were used to remove ligands that were not covalently linked to the target protein. Finally, the DNA associated with the crosslinked peptides was recovered and carried forward to produce the input library for the next round of selection. We performed five rounds of selection and after observing significant enrichment in round five, next generation sequencing was performed on the recovered DNA libraries (Supplementary Figure S5, Table S1). Encouragingly, sequencing results after each round indicated a clear increase in the proportion of Bpacontaining sequences in the top 100 sequences (Figure $2 \mathrm{~b}$ ), suggesting that non-covalent ligands were effectively depleted as the selection progressed. After only the first round of selection, we observed a significant improvement to $52 \%$ of sequences containing at least one $\mathrm{Bpa}$, which further improved to reach $100 \%$ after round four.

Sequence alignments of the top 500 sequences from the final selection round then allowed us to identify consensus peptide sequences that were divided into distinct families. To validate the identified sequences, we selected two of the most abundant hits, Bpa-P1, Bpa-P2, which accounted for $32 \%$ and $5 \%$ of the total sequencing reads recovered in round 5 respectively (Figure $2 c$ and d). We synthesised both unlabelled (Bpa-P1, Bpa-P2) and fluorescently tagged (Bpa-P1-FAM, Bpa-P2-FAM) versions of these hits for analysis by a variety of assays. For Bpa-P1-FAM and Bpa-P2-FAM the 5carboxyfluorescein label was installed at the $C$-terminus through coupling onto the sidechain of an additional lysine residue, using a selective protecting group strategy.

Binding affinities for the two fluorescently-tagged peptides were then determined using a direct fluorescence anisotropy assay (Figure 2d, Supplementary Figure S6). Interestingly the binding affinities (Bpa-P1-FAM: $29 \pm 10 \mu \mathrm{M}$, Bpa-P1-FAM: $14 \pm 1 \mu \mathrm{M}$ ) were substantially weaker than is usually observed from a standard RaPID selection, where (sub-)nanomolar affinities are frequently observed. ${ }^{27,31,32}$ Having established that our peptides were able to bind to BRD3-BD2, we next assessed the crosslinking efficiency of the unlabelled variants. We were pleased to find that both peptides efficiently crosslinked to BRD3-BD2 upon exposure to UV light. After incubation with two equivalents of peptide and irradiation at $365 \mathrm{~nm}$ for only 5 minutes, we observed around $40 \%$ protein modification as analysed by sample separation on an SDS-PAGE gel and Coomassie staining. As expected, longer UV exposure led to higher protein modification, reaching 96\% for Bpa-P1 and 78\% for Bpa-P2 after 40 minutes (Figure $3 b, 3 c$ ). This superior crosslinking ability of Bpa-P1 corresponded well with its higher sequence enrichment in the selection, though it contrasts with the slightly weaker binding affinity we determined. This discrepancy may be explained by the Bpa moiety in Bpa-P1 being more optimally positioned for protein crosslinking than in Bpa-P2. Mass spectrometry was then used to confirm the protein modification observed was due to the addition of a single peptide molecule. Following 
incubation of BRD3-BD2 with each peptide we observed a mass shift corresponding to addition of a single peptide (Figure 3d).

Next we explored the specificity of our peptides by testing their ability to crosslink to other closely related bromodomains in the BET-family, which in addition to BRD3 consists of three other conserved members (BRD2, BRD4 and BRDT) each possessing a pair of tandem bromodomains. ${ }^{28,33}$ Interestingly, despite their high sequence similarities, we found that the peptide ligands crosslinked more efficiently to BRD3-BD2 than to the closely related BRD2-BD2 and BRD4-BD2. Further, no crosslinking was observed to the $N$-terminal BD of BRD3 (BRD3-BD1) (Figure 3e). Similar to results with BRD3-BD2, across the proteins that showed covalent modification, Bpa-P1 crosslinked to a greater extent than Bpa-P2.

To further assess the selectivity of the crosslinking ligands, we first tested whether our FAM labelled peptides could selectively label purified recombinant BRD3-BD2 when mixed with BSA (Figure 4a). After exposure to UV light for 1 hour, both peptides selectively labelled BRD3-BD2 in the presence of BSA as observed by in-gel fluorescence of the FAM label after separation by SDS-PAGE. Control experiments were carried out without irradiation where no labelling was observed. Next, we evaluated labelling in cell lysate (HEK 293T) that had been spiked with purified recombinant BRD3BD2 (Figure 4b). Pleasingly, we observed high levels of selectivity at a range of ligand concentrations $(4,2,1 \mu \mathrm{M})$. Even at the highest concentration tested, we did not see any significant off-target background labelling. Consistent with our earlier experiments with the untagged peptides, these crosslinking experiments using the labelled peptides show Bpa-P1 is a moderately better crosslinker than Bpa-P2. Together the data with purified proteins and in lysate suggest that our peptides are highly selective for their protein target.

\section{Conclusion}

In conclusion, we have developed XL-RaPID, a generally applicable strategy to isolate photoactivatable ligands to a target protein of interest. Application of XL-RaPID to BRD3-BD2 resulted in $p$ Bpacontaining cyclic peptide ligands that efficiently and selectively photocrosslinked with their target, even in the context of complex cell lysates. Our peptides showed substantially weaker binding affinities than is usually found in RaPID screens, suggesting that crosslinker positioning is likely more important than affinity, thus highlighting the power of selecting directly for photocrosslinking efficiency rather than retrofitting a crosslinking moiety to a known ligand. In the future, the same approach could be used to produce photocrosslinking ligands containing other warheads compatible with in vitro translation, such as diazirines. ${ }^{34-37}$ Further we envisage that this same modified affinity 
panning protocol could be applied more widely to the discovery of covalent cyclic peptide ligands from mRNA display libraries incorporating reactive covalent warheads.

\section{Data Availability}

Detailed experimental protocols, sequencing data and Supplementary Figures are provided in the ESI.

\section{Conflict of Interest}

The authors declare no conflict of interest.

\section{Acknowledgements}

We would like to thank the Crick Advanced Sequencing Science Technology Platform for assistance with next generation sequencing and the Crick Structural Biology Science Technology Platform for expert technical support and access to plate readers. We would also like to thank Lisa Haigh (Imperial College London) for assistance with MALDI mass spectrometry. This work was supported by the Francis Crick Institute which receives its core funding from Cancer Research UK (FC001748), the UK Medical Research Council (FC001748), and the Wellcome Trust (FC001748). For the purpose of Open Access, the authors have applied a CC BY public copyright licence to any Author Accepted Manuscript version arising from this submission.

\section{References}

1 W. Zhao, A. R. Cross, C. Crowe-McAuliffe, A. Weigert-Munoz, E. E. Csatary, A. E. Solinski, J. Krysiak, J. B. Goldberg, D. N. Wilson, E. Medina, W. M. Wuest and S. A. Sieber, Angew. Chemie - Int. Ed., 2019, 58, 8581-8584.

2 J. J. Hulce, A. B. Cognetta, M. J. Niphakis, S. E. Tully and B. F. Cravatt, Nat. Methods, $2013,10,259-264$.

3 J. Eirich, R. Orth and S. A. Sieber, J. Am. Chem. Soc., 2011, 133, 12144-12153.

$4 \quad$ N. R. Burton, P. Kim and K. M. Backus, Org. Biomol. Chem., 2021, 19, 7792-7809.

5 E. Smith and I. Collins, Future Med. Chem., 2015, 7, 159-183.

6 T. Seifert, M. Malo, J. Lengqvist, C. Sihlbom, E. M. Jarho and K. Luthman, J. Med. Chem., 2016, 59, 10794-10799.

7 T. A. Martin, C. T. Herman, F. T. Limpoco, M. C. Michael, G. K. Potts and R. C. Bailey, 
ACS Appl. Mater. Interfaces, 2011, 3, 3762-3771.

8 X. Li, J. H. Cao, Y. Li, P. Rondard, Y. Zhang, P. Yi, J. F. Liu and F. J. Nan, J. Med. Chem., 2008, 51, 3057-3060.

9 T. M. Ayele, S. D. Knutson, S. Ellipilli, H. Hwang and J. M. Heemstra, Bioconjug. Chem., 2019, 30, 1309-1313.

10 F. Kotzyba-Hibert, I. Kapfer and M. Goeldner, Angew. Chemie Int. Ed., 1995, 34, 12961312.

11 D. P. Murale, S. C. Hong, M. M. Haque and J. S. Lee, Proteome Sci., 2017, 15, 1-34.

12 M. M. Hassan and O. O. Olaoye, Molecules, , DOI:10.3390/molecules25102285.

13 L. Dubinsky, B. P. Krom and M. M. Meijler, Bioorganic Med. Chem., 2012, 20, 554570.

14 G. Dormán, H. Nakamura, A. Pulsipher and G. D. Prestwich, Chem. Rev., 2016, 116, 15284-15398.

15 R. E. Galardy, L. C. Craig, J. D. Jamieson and M. P. Printz, J. Biol. Chem., 1974, 249, 3510-3518.

16 J. W. Chin, A. B. Martin, D. S. King, L. Wang and P. G. Schultz, Proc. Natl. Acad. Sci. U. S. A., 2002, 99, 11020-11024.

17 I. S. Farell, R. Toroney, J. L. Hazen, R. A. Mehl and J. W. Chin, Nat. Methods, 2005, 2, 377-384.

18 Y. Wu, L. B. Olsen, Y. H. Lau, C. H. Jensen, M. Rossmann, Y. R. Baker, H. F. Sore, S. Collins and D. R. Spring, ChemBioChem, 2016, 17, 689-692.

19 F. Tzakoniati, H. Xu, T. Li, N. Garcia, C. Kugel, J. Payandeh, C. M. Koth and E. W. Tate, Cell Chem. Biol., 2020, 27, 306-313.e4.

20 R. Obexer, L. J. Walport and H. Suga, Curr. Opin. Chem. Biol., 2017, 38, 52-61.

21 S. Bellotto, S. Chen, I. Rentero Rebollo, H. A. Wegner and C. Heinis, J. Am. Chem. Soc., 2014, 136, 5880-5883. 
22 S. Chen, S. Lovell, S. Lee, M. Fellner, P. D. Mace and M. Bogyo, Nat. Biotechnol., 2021, 39, 490-498.

23 Y. Huang, M. M. Wiedmann and H. Suga, Chem. Rev., 2019, 119, 10360-10391.

24 H. Suga, J. Pept. Sci., 2018, 24, 1-7.

25 H. Saito, D. Kourouklis and H. Suga, 2001, 20, 1797-1806.

26 H. Murakami, D. Kourouklis and H. Suga, Chem. Biol., 2003, 10, 1077-1084.

27 K. Patel, L. Walport, J. Walshe, P. Solomon, J. Low, D. Tran, K. Mouradian, A. Silva, L. Wilkinson-White, J. Matthews, J. Mitchell Guss, R. Payne, T. Passioura, H. Suga and J. Mackay, Proc. Natl. Acad. Sci., 2020, 117, 26728-26738.

28 N. Wang, R. Wu, D. Tang and R. Kang, Signal Transduct. Target. Ther., , DOI:10.1038/s41392-020-00384-4.

29 T. Fujisawa and P. Filippakopoulos, Nat. Rev. Mol. Cell Biol., 2017, 18, 246-262.

30 S. C. Hsu and G. A. Blobel, Cold Spring Harb. Symp. Quant. Biol., 2017, 82, 37-43.

31 E. Stefan, R. Obexer, S. Hofmann, K. Vu Huu, Y. Huang, N. Morgner, H. Suga and R. Tampé, Elife, 2021, 10, 1-24.

32 J. M. Rogers, M. Nawatha, B. Lemma, G. B. Vamisetti, I. Livneh, U. Barash, I. Vlodavsky, A. Ciechanover, D. Fushman, H. Suga and A. Brik, RSC Chem. Biol., 2021, 2, $513-522$.

33 Y. Taniguchi, Int. J. Mol. Sci., , DOI:10.3390/ijms17111849.

34 M. Vila-Perellö, M. R. Pratt, F. Tulin and T. W. Muir, J. Am. Chem. Soc., 2007, 129, 8068-8069.

35 T. Yang, X.-M. Li, X. Bao, Y. M. E. Fung and X. D. Li, Nat. Chem. Biol., 2015, 12, 70-72.

36 D. He, X. Xie, F. Yang, H. Zhang, H. Su, Y. Ge, H. Song and P. R. Chen, Angew Chem Int Ed Engl, 2017, 56, 14521-14525.

37 J. Lin, X. Bao and X. D. Li, Mol. Cell, 2021, 81, 2669-2681.e9. 
Figure 1

Previous work:

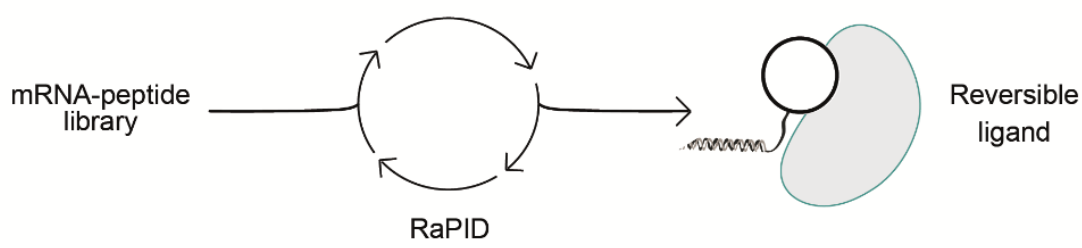

This work:

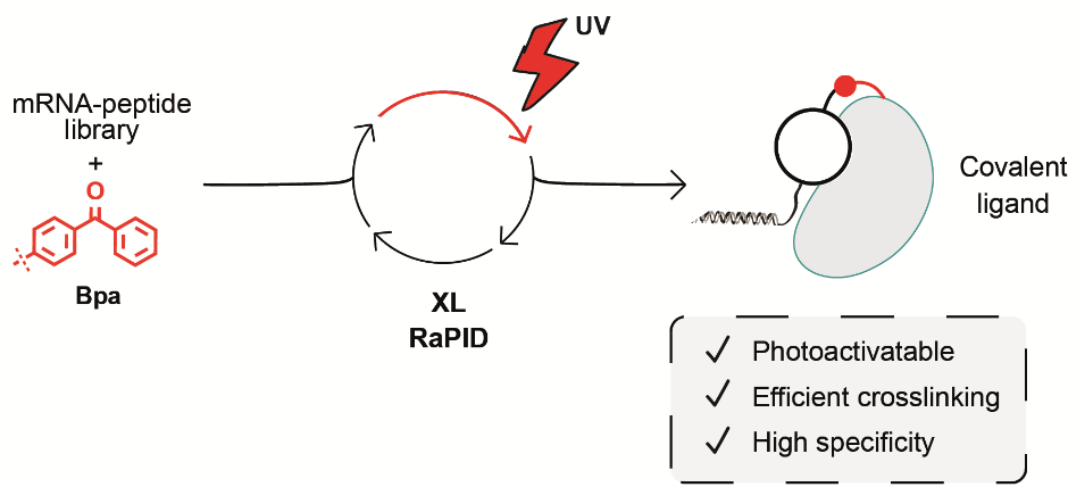

Figure 1. Comparison between standard and crosslinking mRNA display. 
Figure 2

a

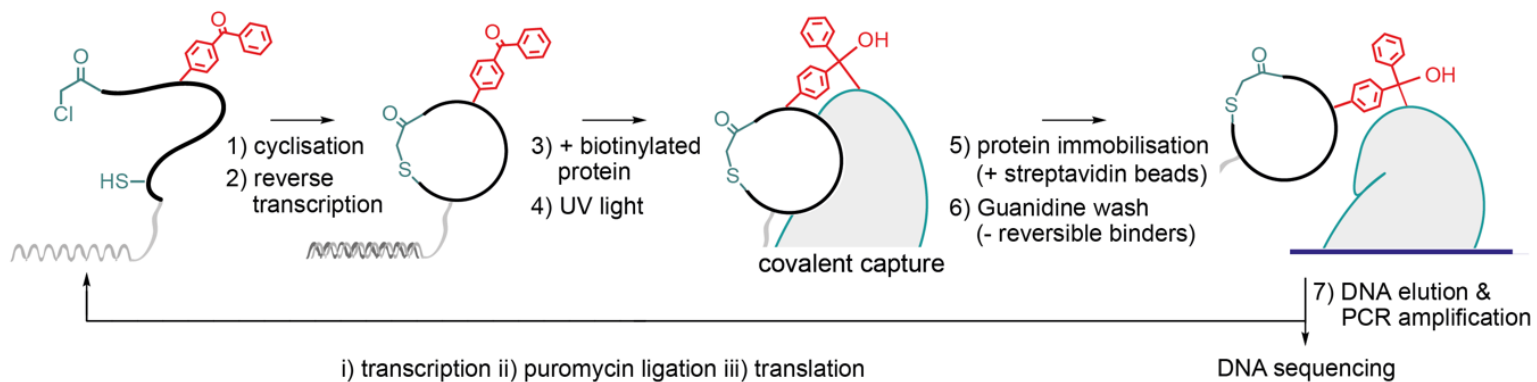

b

C
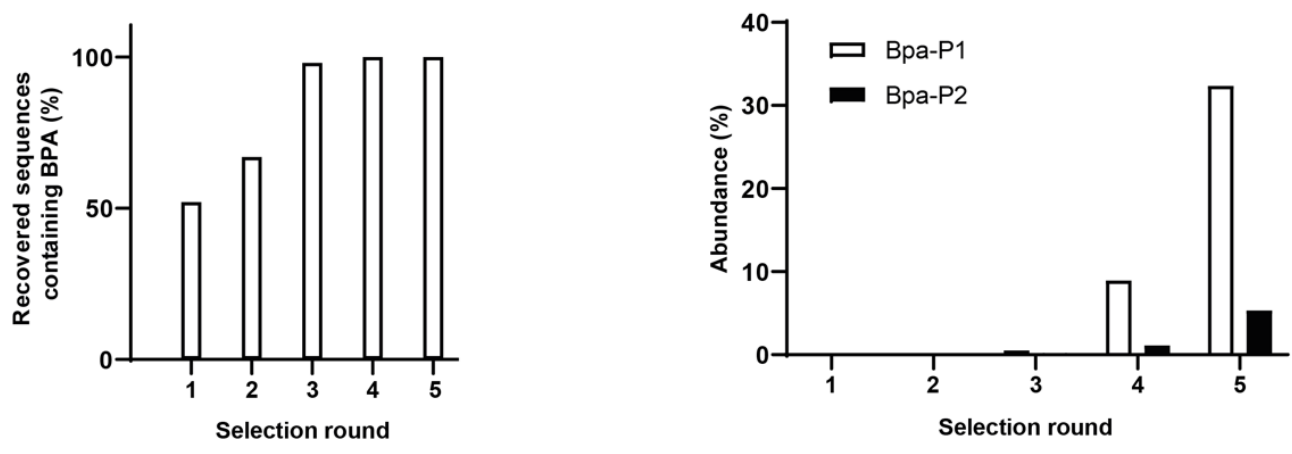

d

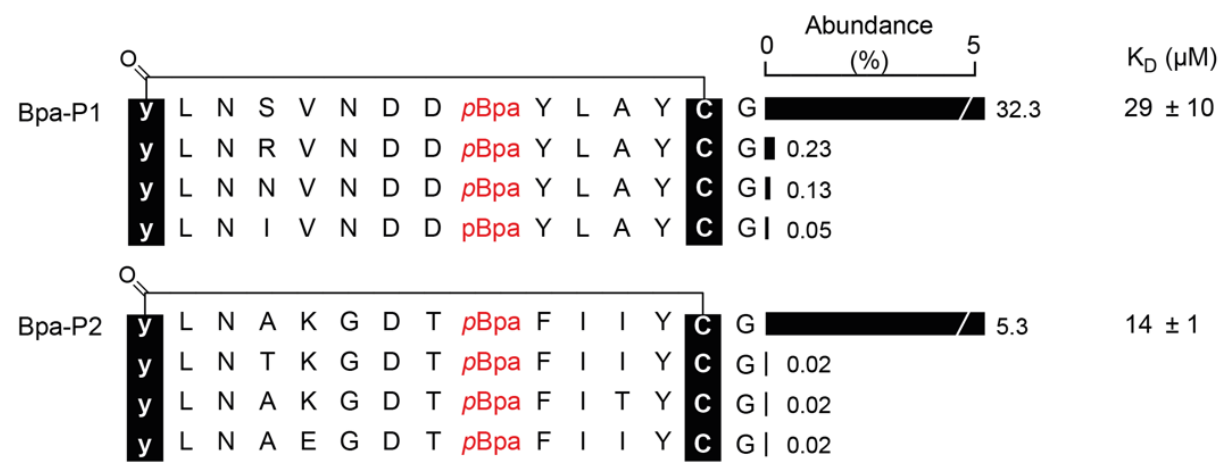

Figure 2. mRNA display-based selection of photocrosslinking peptide ligands. a, Schematic depiction of the XLRaPID selection strategy. $\mathbf{b}$, Percentage of sequences recovered after each round of selection that contains at least one Bpa (calculated using the top 100 sequences). c, Percentage abundance of the top two hits (Bpa-P1, Bpa-P2) after each round of selection. $\mathbf{d}$, Selected peptide sequences recovered after five rounds of selection. Binding affinities were determined by direct fluorescence anisotropy using FAM labelled peptides and are represented as the mean of at least three replicates \pm standard deviation. 
Figure 3

a

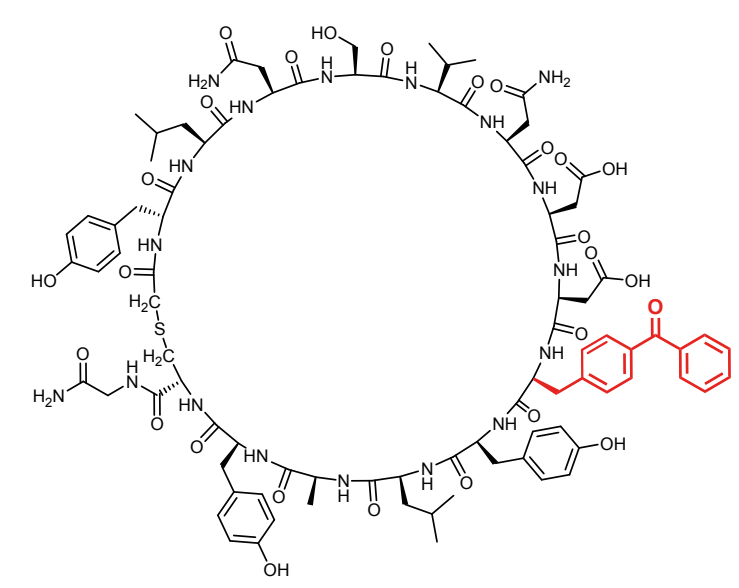

Bpa-P1

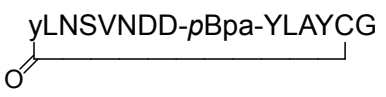

b

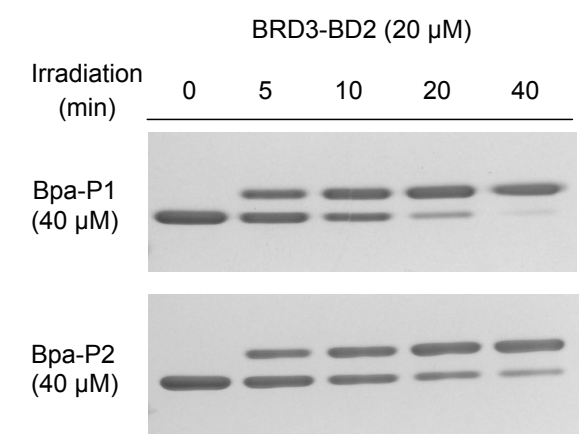

d

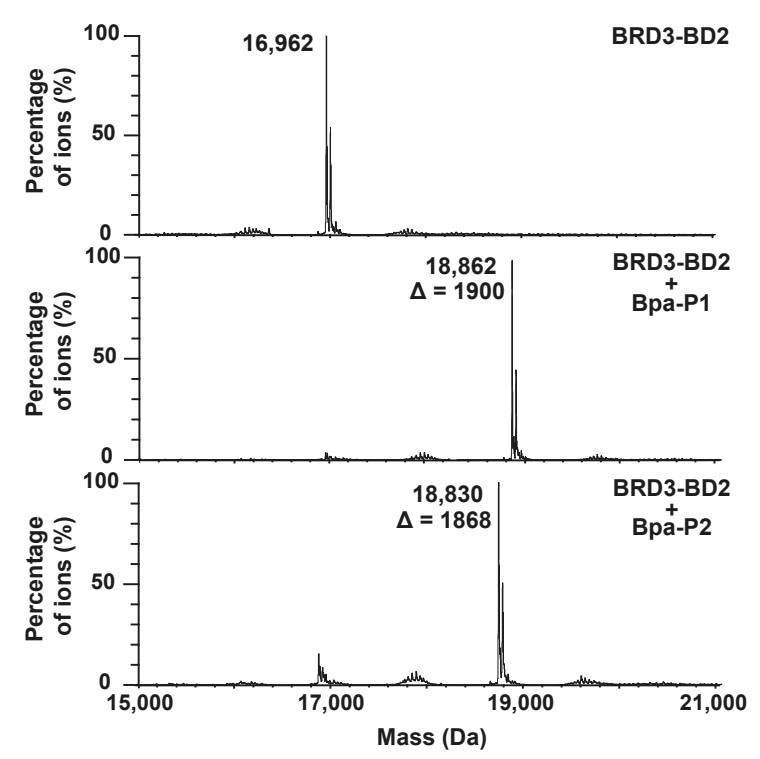

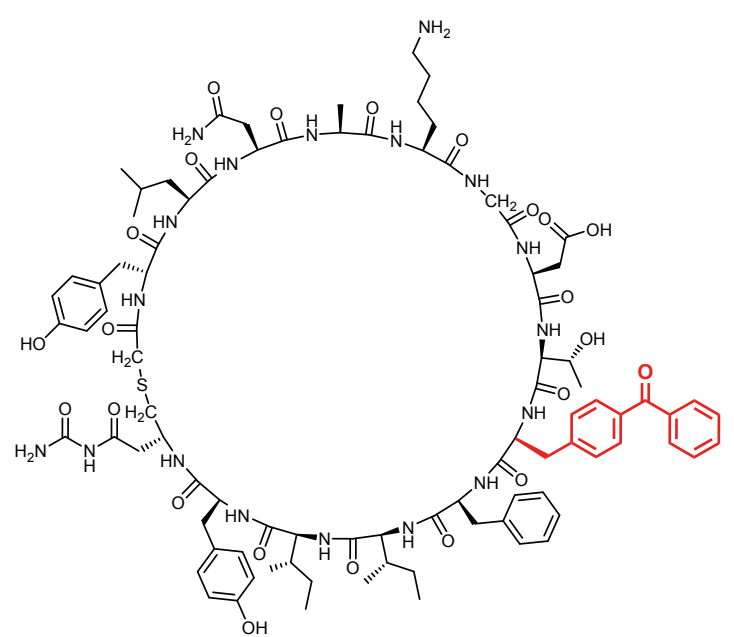

Bpa-P2

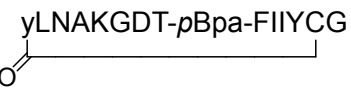

C

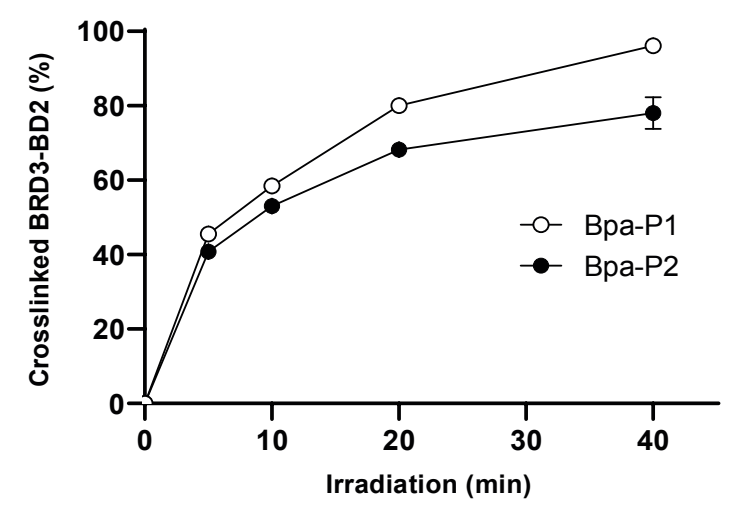

e

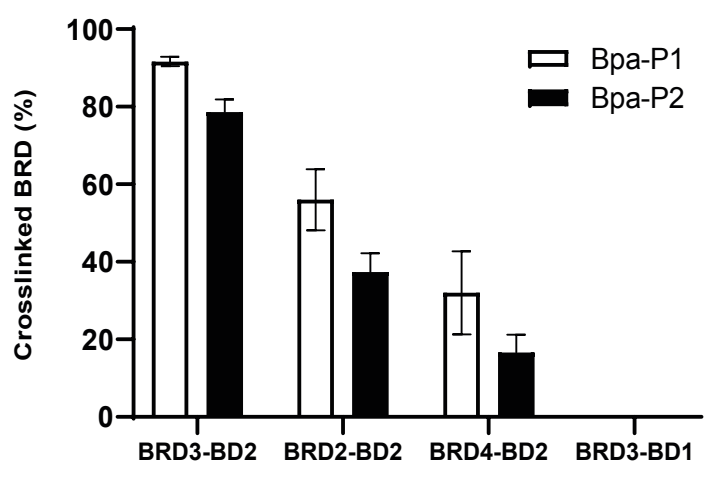


Figure 3. Photocrosslinking of BRDs with Bpa-containing ligands. a, Chemical structures of cyclic peptides BpaP1, Bpa-P2. b, Crosslinking between BRD3-BD2 $(20 \mu \mathrm{M})$ and cyclic peptides $(40 \mu \mathrm{M})$ after exposure to UV irradiation (365 nm) for variable amounts of time $(0,5,10,20,40 \mathrm{~min})$. Samples were separated on an SDS-PAGE gel and visualised by Coomassie staining. c, Plot of percentage of crosslinked BRD3-BD2 calculated by densitometric analysis of protein bands from b. Crosslinking efficiencies are represented as the mean of triplicate experiments \pm 1 standard deviation. $\mathbf{d}$, Confirmation of photocrosslinking of BRD3-BD2 by mass spectrometry. A mixture of BRD3-BD2 $(2 \mu \mathrm{M})$ and cyclic peptides $(20 \mu \mathrm{M})$ were exposed to UV irradiation for 30 min and analysed by LCMS. The mass shifts correspond to adducts formed from the addition of Bpa-P1 or BpaP2. e, Crosslinking of BRD3-BD2/BRD2-BD2/BRD4-BD2/BRD3-BD1 (2 $\mu \mathrm{M})$ by cyclic peptides (20 $\mu \mathrm{M})$ after exposure to UV irradiation for $30 \mathrm{~min}$. Samples were analysed and processed as described in b/c. Crosslinking efficiencies are represented as the mean of triplicate experiments \pm 1 standard deviation. 
Figure 4

a

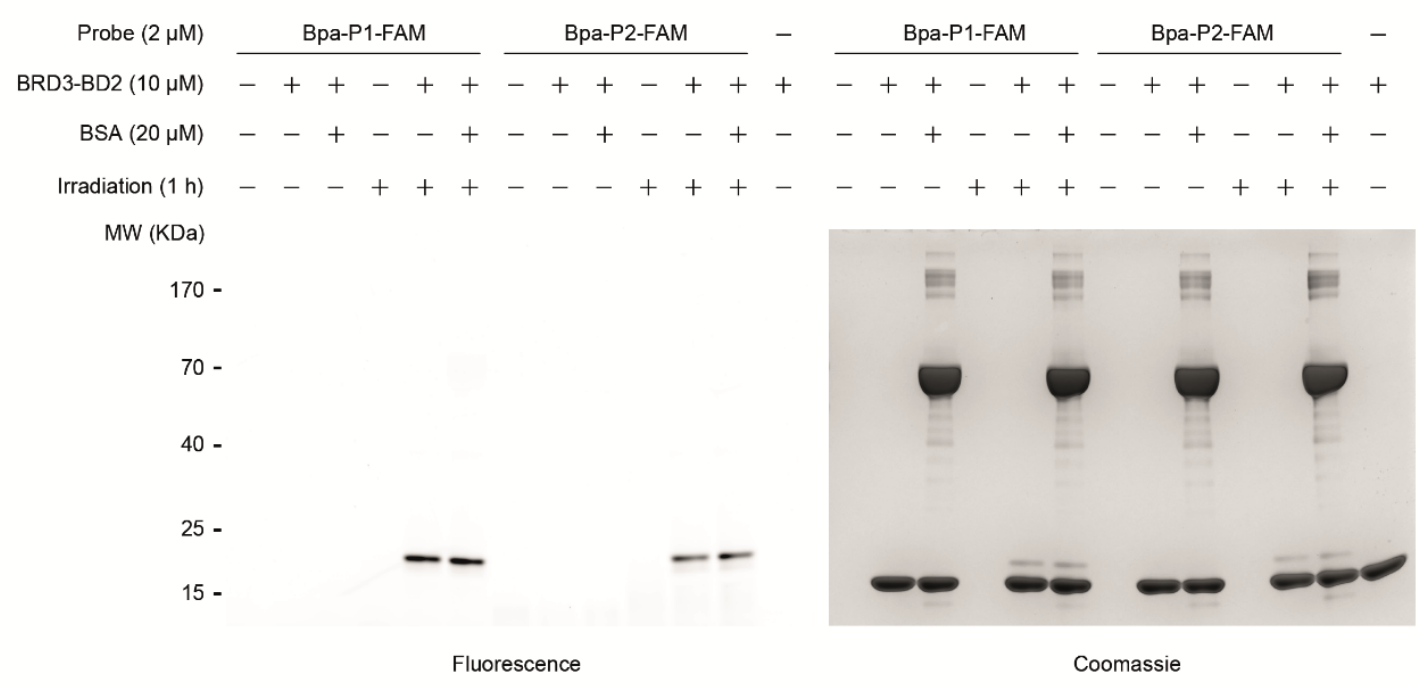

b

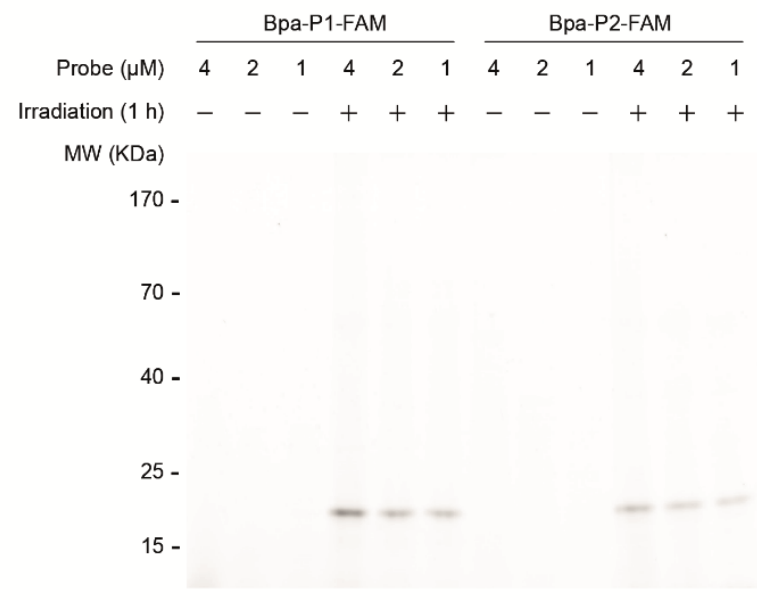

Fluorescence

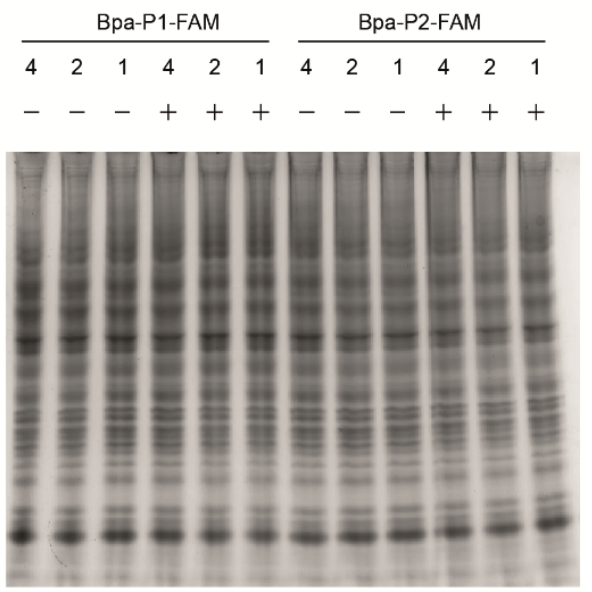

Coomassie

Figure 4. FAM cyclic peptides selectively label BRD3-BD2 in complex mixtures. a, Mixtures containing BRD3-BD2

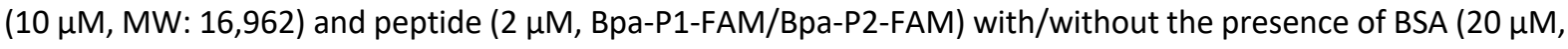
MW: 66,000) were irradiated at $365 \mathrm{~nm}$ for $1 \mathrm{~h}$. Samples were separated on an SDS-PAGE gel and visualised by in-gel fluorescence and Coomassie staining. Control experiments were performed without UV irradiation. $\mathbf{b}$, Photoaffinity labelling of BRD3-BD2 (1 $\mu \mathrm{M})$ spiked into cell lysate (450 $\mu \mathrm{g}$, HEK 293T cells). BRD3-BD2 was preferentially labelled by various concentrations of peptide $(4,2$ or $1 \mu \mathrm{M})$ after UV exposure (1 h). Control experiments were performed without UV irradiation. Samples were analysed as described in a. 